\title{
ATENCIÓN PLENA: LA RELACIÓN ENTRE LA ACEPTACIÓN DE LAS EMOCIONES Y LA SATISFACCIÓN CON LA VIDA EN ESTUDIANTES PREGRADUADOS
}

\author{
Alberto Paramio Leiva \\ alparamiole@gmail.com \\ Daniel Cruz Jurado \\ Antonio Zayas García \\ Paloma Gil-Olarte Márquez \\ Rocío Guil Bozal \\ Universidad de Cádiz
}

Fecha de Recepción: 8 Marzo 2018

Fecha de Admisión: 10 Abril 2018

\section{RESUMEN}

El uso de las Terapias de Tercera Generación se ha intensificado en todos los ámbitos de la psicología relacionados con la salud. La Atención plena (Mindfulness) se ha postulado como una de las principales opciones elegidas de entre los profesionales, volviéndose una práctica de uso ascendente en el ejercicio clínico y el desarrollo de una buena salud psicológica (Ford, Lam, John, \& Mauss, 2017). La satisfacción con la vida es uno de los componentes de la salud psicológica más relacionados con estas terapias. El presente estudio tiene como objetivos: 1. Examinar la posible relación entre los cinco factores de la Atención plena (Observación, Descripción, Actuar con conciencia, No juzgar y No reactividad) y la satisfacción con la vida. 2. Conocer la capacidad predictiva y explicativa de dichos factores respecto a la satisfacción con la vida. Se utilizó para ello una muestra de 53 estudiantes pregraduados (tercero y cuarto de carrera) de la Universidad de Cádiz que realizaron voluntariamente y a los que se les administró de forma telemática los cuestionarios: Five Facet Mindfulness Questionnaire (Aguado et al., 2015) y Satisfaction with Life Scale (Atienza, Pons \& Balaguer, 2000). Los análisis de correlaciones bivariadas realizados mostraron que sólo los factores de la atención plena No juzgar y Actuar con conciencia, relacionaban de forma positiva y estadísticamente significativa con la satisfacción con la vida. El resultado del análisis de regresión simple efectuado reveló que ambos factores explicaban casi un $24 \%$ de la varianza de la satisfacción con la vida (R cuadrado= 0,238 ), si bien solo el factor No Juzgar (aceptación de la experiencia internas y las emociones) mostró una capacidad predictiva positiva estadísticamente significativa ( = $0,394)$. Ello nos aporta evidencia de que podemos incrementar la satisfacción con la vida del alumnado pregraduado a partir del desarrollo de su capacidad de aceptar las experiencias internas y las emociones. 


\section{ATENCIÓN PLENA: LA RELACIÓN ENTRE LA ACEPTACIÓN DE LAS EMOCIONES Y LA SATISFACCIÓN CON LA VIDA EN ESTUDIANTES PREGRADUADOS}

Palabras clave: atención plena; aceptación; satisfacción con la vida; estudiantes pregraduados; terapias de tercera generación

\section{ABSTRACT \\ Mindfulness: the relationship between the acceptance of emotions and satisfaction with life in undergraduate students.}

The use of Third Generation Therapies has intensified in all areas of health-related psychology. Mindfulness has been postulated as one of the main options chosen among professionals, becoming a practice of ascending use in clinical practice and the development of good psychological health (Ford, Lam, John, \& Mauss, 2017). The satisfaction with life is one of the components of psychological health most related to these therapies. The present study aims to: 1. Examine the possible relationship between the five factors of Mindfulness (Observing, Describing, Acting with awareness, Non-judging, and Non-reactivity) and satisfaction with life. 2. Know the predictive and explanatory capacity of these factors with respect to life satisfaction. A sample of 53 undergraduated students (third and fourth degree) from the University of Cádiz was used. They voluntarily completed the questionnaires: Five Facet Mindfulness Questionnaire (Aguado et al., 2015). Satisfaction with Life Scale (Atienza, Pons \& Balaguer, 2000). The analyzes of bivariate correlations made showed that only the factors of mindfulness Non-judging and acting with awareness, related in a positive and statistically significant way to satisfaction with life. The results of the simple regression analysis revealed that both factors accounted for almost $24 \%$ of the variance of life satisfaction (R squared= 0,238 ), although only the Non-judging factor (acceptance of internal experience and emotions) showed a statistically significant positive predictive capacity $(=0,394)$. This gives us evidence that we can increase the satisfaction with the life of the undergraduate students by developing their acceptance for internal experiences and emotions.

Keywords: mindfulness; acceptance; satisfaction with life; undergraduate students; third-generation therapies

\section{ANTECEDENTES}

Las Terapias de Tercera Generación suponen un nuevo enfoque en el ejercicio clínico de la psicología para promover y fomentar la salud (Hayes, 2004). Durante los últimos años, las técnicas relacionadas con las terapias contextuales y de aceptación han incrementado su uso por parte de los profesionales sanitarios como respuesta a las limitaciones de la intervención propiciadas por las terapias tradicionales. A raíz de esta tendencia, se han encontrado mejoras significativas en el tratamiento de los trastornos del estado de ánimo como la ansiedad o la depresión (Aldao et al., 2010), por lo que la investigación ha aumentado el foco sobre los constructos que comportan están técnicas (Eisenlohr-Moul, Peters, \& Baer, 2015). Estas nuevas líneas de investigación están ayudando a explicar y comprender estos constructos y su relación con aquellas variables que comportan una buena salud psicológica (Ciesla, Reilly, Dickson, Emanuel, \& Updegraff, 2012; Mennin \& Fresco, 2013). La idea de la salud psicológica está compuesta por dimensiones psicológicas positivas y negativas, entre las cuales se encuentra la satisfacción con la vida que a su vez se postula como una de las más importantes para determinar la percepción de bienestar en del individuo (Ford, Lam, John, \& Mauss, 2017).

Dentro de estas terapias, la Atención plena o Mindfulness ha ganado importancia en la investigación que ha desarrollado las bases empíricas de la misma desde su concepción a partir de sus raíces en la meditación oriental (Academic Mindfulness Interest Group, 2006). Su estudio sobre el bienestar se ha desarrollado gracias al desarrollado de diferentes instrumentos que tratan de explicar, factorizar y medir sus dimensiones. Entre los más destacados se encuentra el Five Facet 
Mindfulness Questionnaire (FFMQ), compuesto por cinco factores o facetas: Observación, Descripción, Actuar con conciencia, No juzgar y No reactividad. De todos estos, el factor No Juzgar sería el que se correspondería en mayor medida con la aceptación (Jiménez, 2018), constructo fundamental en las Terapias de Tercera Generación que hace referencia a la capacidad para no juzgar la experiencia externa y/o las emociones del individuo. La traducción y validación de dicha escala a la población española se realizó recientemente, lo que posibilita su implementación en ésta y futuras investigación (Santana \& Ordi, 2016).

La aceptación es uno de éstos constructos que más atención ha recibido por parte de éstas nuevas corrientes de investigación debido a su importancia en estas nuevas terapias, sobretodo en su relación a su importancia a la hora de explicar su efecto sobre las emociones negativas (CampbellSills et al., 2006; Singer \& Dobson, 2007). No obstante, la salud psicológica en su conjunto, incluidas sus dimensiones positivas (resiliencia, satisfacción con la vida, bienestar subjetivo, etc.) están empezando a constituir el núcleo de la investigación de la aceptación (Ford, Lam, John, \& Mauss, 2017). Sin embargo, es necesario seguir trabajando en esta dirección para sentar las bases de la futura intervención psicológica mediante el uso de las técnicas de estas terapias.

La muestra de estudiantes pregraduados (considerando los mismos como aquellos alumnos que se encuentran en tercero y cuarto de carrera) por motivo de su relación de altos niveles de aceptación hacia estresores múltiples, se posiciona como una población atractiva de cara a futuras investigaciones (Ostafin et al., 2014). Los últimos estudios realizado en este sector de las poblaciones han sido cruciales para la determinación de las dimensiones más importantes de la salud psicológica (Almeida, 2005).

El presente estudio piloto pretende conocer la relación entre los diferentes factores del FFMQ y la satisfacción con la vida, como un primer paso para conocer la importancia de la aceptación en la salud psicológica en los estudiantes pregraduados de la Universidad de Cádiz. La importancia del resto de dimensiones y las relaciones con otros constructos de las Terapias de Tercera Generación, todavía están son objetivos pendientes de estudio en esta población.

\section{OBJETIVOS}

El principal objetivo de nuestro estudio es comprobar la relación existente entre los cinco factores de la Atención plena con la satisfacción con la vida para sentar precedente acerca de la importancia de estudiar dichos factores con los diferentes componentes que determinan la salud psicológica en los alumnos pregraduados.

Examinar la posible relación entre los cinco factores de la Atención plena (Observación, Descripción, Actuar con conciencia, No juzgar y No reactividad) y la satisfacción con la vida.

Conocer la capacidad predictiva y explicativa de dichos factores respecto a la satisfacción con la vida.

\section{MUESTRA DEL ESTUDIO}

El estudio fue realizado por el Equipo de investigación de inteligencia emocional de la Universidad de Cádiz sobre una muestra de 53 estudiantes de los cursos tercero y cuarto de sus respectivos grados, que cumplimentaron de forma telemática y voluntaria los cuestionarios. Todos ellos residían en la provincia de Cádiz y la edad media se situaba en los 24 años.

\section{METODOLOGÍA E INSTRUMENTOS}

Las pruebas fueron suministradas telemáticamente a través del correo electrónico de los participantes y rellenadas a través de la plataforma on-line Google Forms. Estos instrumentos fueron seleccionados en base a los estudios anteriores que relacionaban la Atención plena con distintos 


\section{ATENCIÓN PLENA: LA RELACIÓN ENTRE LA ACEPTACIÓN DE LAS EMOCIONES Y LA SATISFACCIÓN CON LA VIDA EN ESTUDIANTES PREGRADUADOS}

factores de la salud psicológica (Ford, Lam, John, \& Mauss, 2017). En el presente estudio, la variable satisfacción con la vida fue la elegida para comprobar si la relación se replicaba en la muestra seleccionada, desestimando otras variables para estudios posteriores que puedan sustentarse en los resultados de este último.

Escala de Satisfacción con la Vida (Atienza, Pons \& Balaguer, 2000). Instrumento breve que nos permite evaluar los juicios cognitivos relacionados con la satisfacción con la vida y la satisfacción personal.

Escala Five Facet Mindfulness Questionnaire (Aguado et al., 2015). Cuestionario que examina los diferentes factores que comportan la Atención plena (Observación, Descripción, Actuar con conciencia, No juzgar y No reactividad), así como una puntuación global de la toma de consciencia en el presente.

Observación: Implica la habilidad de percibir, reconocer y sentir estímulos internos o externos. Conlleva el estar abierto y perceptivo para reconocer estímulos que normalmente no están en el objeto principal de la atención y que permanecen al margen de la acción principal, por ser comunes 0 cotidianos.

Descripción: Hace referencia a describir o etiquetar con palabras la experiencia que se vive relacionada con la atención plena. Implica también la capacidad de encontrar palabras para expresar el contenido atencional observado; expresando emociones, sentimientos, estados de ánimo o estímulos sensoriales o sensitivos.

Actuar con conciencia: Implica el actuar con conciencia al realizar actos en los que se está concentrado o se toma conciencia de aspectos específicos de la acción. Es contrario al comportamiento en "piloto automático", es decir actuar sin ser consciente de ello.

No juzgar: Se refiere a tener una visión ecuánime ante los pensamientos, sensaciones o emociones que se perciben. Estos son observados como objetos temporales que surgen en el campo perceptivo, y son percibidos sin identificarse, apegarse o rechazarlos.

No reactividad: Esta actitud conlleva distanciarse y no reaccionar durante un breve lapso de tiempo al estímulo percibido para poder valorar y llevar a cabo una mejor reacción.

Para la gestión y el análisis de los datos recolectados, se ha utilizado el software informático IBM SPSS Statistics 24.

\section{RESULTADOS}

Los primeros resultados (Tabla 1), se presenta las intercorrelaciones entre las variables satisfacción con la vida y los cinco factores que conforman la Atención plena. Sólo los factores Actuar con conciencia y No juzgar mostraron una relación estadísticamente significativa con la satisfacción con la vida. Esta relación positiva entre las variables supondría que el aumento o disminución en los niveles de cada uno de estos factores, se vería acompañado de la modificación de la satisfacción con la vida en el mismo sentido.

Tabla 1. Análisis de correlaciones entre la satisfacción con la vida y los factores del FFMQ.

\begin{tabular}{llllll} 
& Observación & Descripción & ActuarConc & NoJuzgar & NoReactividad \\
\hline SatisfVida &, 195 &, 011 &, $331^{*}$ &, $463^{* *}$ &, 257 \\
\hline *. La correlación es significativa en el nivel 0,05 (bilateral). & & \\
**. La correlación es significativa en el nivel 0,01 (bilateral). & &
\end{tabular}

A continuación, para comprobar qué factores del FFMQ explican y/o predicen la satisfacción con la vida en la muestra estudiada se realiza una regresión lineal con aquellos factores que previamente habían mostrado una correlación estadísticamente significativa. 
Tabla 2.1. Resumen del modelo de regresión sobre la satisfacción con la vida.

\begin{tabular}{lllll} 
Modelo & $\mathrm{R}$ & R cuadrado & R cuadrado ajustado & $\begin{array}{l}\text { Error estándar de la } \\
\text { estimación }\end{array}$ \\
\hline 1 &, $488^{\mathrm{a}}$ &, 238 &, 208 & 1,08952 \\
\hline
\end{tabular}

a. Predictores: (Constante), NoJuzgar, ActuarConc

Tabla 2.2. Análisis ANOVA de la regresión lineal sobre la satisfaccion con la vidad.

\begin{tabular}{llllllll} 
Modelo & & $\begin{array}{l}\text { Suma de } \\
\text { cuadrados }\end{array}$ & gl & Media cuadrática & F & Sig. \\
\hline 1 & Regresión & 18,570 & 2 & 9,285 & 7,822 &, $001^{\mathrm{b}}$ \\
\cline { 2 - 7 } & Residuo & 59,353 & 50 & 1,187 & & \\
\hline & Total & 77,923 & 52 & & & \\
\hline
\end{tabular}

a. Variable dependiente: SatisfVida

b. Predictores: (Constante), NoJuzgar, ActuarConc

Tabla 2.3. Coeficientes del análisis de regresión sobre la depresión.

\begin{tabular}{|c|c|c|c|c|c|c|}
\hline \multirow{2}{*}{\multicolumn{2}{|c|}{ Modelo }} & \multicolumn{2}{|c|}{ Coeficientes no estandarizados } & \multirow{2}{*}{$\begin{array}{l}\text { Coeficientes } \\
\text { estandarizados } \\
\text { Beta }\end{array}$} & \multirow[b]{2}{*}{$\mathrm{t}$} & \multirow[b]{2}{*}{ Sig. } \\
\hline & & $\mathrm{B}$ & Error estándar & & & \\
\hline \multirow[t]{3}{*}{1} & (Constante) & 2,416 &, 556 & & 4,343 &, 000 \\
\hline & ActuarConc & ,216 &, 174 &, 168 & 1,243 &, 220 \\
\hline & NoJuzgar & ,449 &, 154 &, 394 & 2,910 &, 005 \\
\hline
\end{tabular}

a. Variable dependiente: SatisfVida

El análisis de regresión por pasos dio como resultado un modelo significativo (Tabla 2.2), capaz de explicar en un $23,8 \%$ de la varianza de la satisfacción con la vida (R cuadrado $=0,238$ ) (Tabla 2.1). El factor No juzgar resultó como la única variable predictiva estadísticamente significativa del modelo $(=0,394)$ (Tabla 2.3). Por lo que podemos concluir que el factor No juzgar sería el más importante a tener en cuenta para procurar un cambio y, por tanto, producir una mejora, en los niveles de satisfacción con la vida de los estudiantes pregraduados.

\section{DISCUSIÓN}

La aceptación se ha postulado como una de las variables más relevantes en el estudio de las Terapias de Tercera Generación. Los resultados obtenidos a partir de la muestra de nuestra investigación confirman lo que parece una tendencia creciente en la literatura. El factor No juzgar, correspondiente al constructo aceptación, resultó el mejor a la hora de explicar y predecir la varianza de la satisfacción con la vida, como ha ocurrido en investigaciones anteriores (Ford, Lam, John, \& Mauss, 2017), Otros factores, como Actuar con conciencia, que han demostrado ser igualmente eficaces a la hora de explicar y predecir dimensiones de la salud psicológica en otros estudios (Thompson \& Waltz, 2010; Vujanovic et al., 2009), no han resultado significativos en nuestra muestra de pregraduados, sin embargo, pese a la limitación en cuanto variables predictivas, el porcentaje explicativo del modelo obtenido se considera aceptable $(23,8 \%$ de la varianza) y relevante para proseguir con esta línea de investigación. Por lo que, si queremos realizar una intervención que mejore la satisfacción con la vida de los estudiantes pre graduados empleando técnicas relacionadas con la Atención plena, el hecho de aprender a no juzgar y aceptar las emociones y la experiencia resultará determinante. Aunque somos conscientes de que la muestra es limitada, más teniendo en cuenta la especificidad de la población de estudio, los indicios que se evidencian como estudio piloto pretenden sentar las bases para futuras investigadores. 


\section{ATENCIÓN PLENA: LA RELACIÓN ENTRE LA ACEPTACIÓN DE LAS EMOCIONES Y LA SATISFACCIÓN CON LA VIDA EN ESTUDIANTES PREGRADUADOS}

Por otra parte, muchas de las dimensiones componentes psicológicas de la salud psicológica no han sido estudiadas en esta investigación, teniendo muchas de ellas, especialmente las negativas (ansiedad, depresión, supresión emocional), una estrecha relación explicativa con diversos factores de la escala FFMQ (Ford, Lam, John, \& Mauss, 2017) y otros de los constructos principales relacionados con las técnicas empleadas en las Terapias de Tercera Generación. Por tanto, es necesario remarcar la importancia de estudiar los niveles de estas dimensiones en muestras compuestas por pregraduados españoles, así como sus relaciones con los constructos mencionados. En la población española, aún sigue pendiente el aumento del uso de la escala, tanto en clínica como investigación, traducida y validada por Aguado et al. Recientemente (2015), para poder contrastar su eficacia como ha ocurrido con el instrumento original.

Este reto supone un aliciente para la investigación de estas terapias en nuestro país, para que se puedan replicar los resultados obtenidos fuera de nuestras fronteras y comprobar si la aceptación de la experiencia interna y las emociones (No juzgar) supone una variable de peso en la intervención mediante el uso de la Atención plena. Los estresores situacionales que deben afrontar los estudiantes, en especial aquellos que se encuentran en los últimos años de carrera, y su capacidad para sobrellevar y sobreponerse a ellos, los colocan como una muestra de gran interés en el futuro de este tipo de estudios (Michalak et al., 2011), pudiendo sentar precedente para otros estudios con muestras clínicas. También sería de interés incluir variables contextuales, como la situación socioeconómica (Troy, Ford, McRae, Zarolia, \& Mauss, 2016) o el género, variables fundamentales para comprender el bienestar y la satisfacción con la vida en la muestra del estudio.

La necesidad de comprender el papel de la aceptación en la gestión de las emociones en las Terapias de Tercera Generación, es uno de los principales objetivos futuros para avanzar en el desarrollo de técnicas que fomenten la salud psicológica y nos ayude a conocer los mecanismos que actúan sobre las dimensiones responsables de los trastornos del estado de ánimo como la ansiedad o la depresión. Por ello, animamos a continuar la investigación en esta dirección.

\section{CONCLUSIÓN}

A raíz de los resultados obtenidos en el estudio podemos concluir que:

Actuar con conciencia y no juzgar son los únicos factores del FFMQ que correlacionan de forma estadísticamente significativa con la satisfacción con la vida en los estudiantes pregraduados de este estudio piloto y, además, lo hacen de forma positiva.

No juzgar resultó la única variable predictiva y explicativa significativa a la hora de determinar el cambio en la varianza de la satisfacción con la vida, por lo que si se pretende lograr una mejora en la satisfacción con la vida de estos estudiantes pregraduados la aceptación de la experiencia interna y las emociones debe ser el factor más trabajado en la intervención con Terapias de Tercera Generación.

Es necesario continuar la investigando en esta población para conocer la relación de las distintas variables que conforman la salud psicológica con los factores de la Atención plena y si No juzgar se sitúa de nuevo como variable más explicativa y predictiva de estas variables. Así como promover estudios sobre otras Terapias de Tercera Generación que incluyan variables contextuales y sociales, como el nivel socioeconómico.

\section{REFERENCIAS}

Academic Mindfulness Interest Group, M., \& Academic Mindfulness Interest Group, M. (2006). Mindfulness-based psychotherapies: a review of conceptual foundations, empirical evidence and practical considerations. Australian and New Zealand Journal of Psychiatry, 40(4), 285-294. 
Aguado, J., Luciano, J. V., Cebolla, A., Serrano-Blanco, A., Soler, J., \& García-Campayo, J. (2015). Bifactor analysis and construct validity of the five facet mindfulness questionnaire (FFMQ) in non-clinical Spanish samples. Frontiers in psychology, 6, 404.

Aldao, A., \& Nolen-Hoeksema, S. (2010). Specificity of cognitive emotion regulation strategies: A transdiagnostic examination. Behaviour research and therapy, 48(10), 974-983.

Almeida, D. M. (2005). Resilience and vulnerability to daily stressors assessed via diary methods. Current Directions in Psychological Science, 14(2), 64-68.

Atienza, F. L., Pons, D., Balaguer, I., \& García Merita, M. (2000). Propiedades psicométricas de la Escala de Satisfacción con la Vida en adolescentes. Psicothema, 12(2).

Campbell-Sills, L., Barlow, D. H., Brown, T. A., \& Hofmann, S. G. (2006). Effects of suppression and acceptance on emotional responses of individuals with anxiety and mood disorders. Behavioral Research and Therapy, 44(9), 1251-1263.

Ciesla, J. A., Reilly, L. C., Dickson, K. S., Emanuel, A. S., \& Updegraff, J. A. (2012). Dispositional mindfulness moderates the effects of stress among adolescents: rumination as a mediator. Journal of Clinical and Child Adolescent Psychology, 41(6), 760-770.

Eisenlohr-Moul, T., Peters, J. R., \& Baer, R. A. (2015). How do mindfulness-based interventions work? Strategies for studying mechanisms of change in clinical research. In Handbook of Mindfulness and Self-Regulation (pp. 155-170). Springer, New York, NY.

Ford, B. Q., Lam, P., John, O. P., \& Mauss, I. B. (2017). The Psychological Health Benefits of Accepting Negative Emotions and Thoughts: Laboratory, Diary, and Longitudinal Evidence. Journal of personality and social psychology.

Hayes, S. C. (2004). Acceptance and Commitment Therapy and the New Behavior Therapies: Mindfulness, Acceptance, and Relationship.

Jiménez, R. V. M. (2018). La evitación experiencial y la autoestima como factores de vulnerabilidad psicosocial en los trastornos emocionales: ¿variables independientes o relacionadas? (Doctoral dissertation, Universidad de Sevilla).

Michalak, J., Teismann, T., Heidenreich, T., Ströhle, G., \& Vocks, S. (2011). Buffering low selfesteem: The effect of mindful acceptance on the relationship between self-esteem and depression. Personality and Individual Differences, 50(5), 751-754.

Mennin, D. S., \& Fresco, D. M. (2013). What, me worry and ruminate about DSM-5 and RDoC? The importance of targeting negative self-referential processing. Clinical Psychological Science and Practice, 20(3), 258-267.

Ostafin, B. D., Brooks, J. J., \& Laitem, M. (2014). Affective reactivity mediates an inverse relation between mindfulness and anxiety. Mindfulness, 5(5), 520-528.

Sancho, M., De Gracia, M., Rodríguez, R. C., Mallorquí-Bagué, N., Sánchez-González, J., Trujols, J., ... \& Menchón, J. M. (2018). Mindfulness-Based Interventions for the Treatment of Substance and Behavioral Addictions: A Systematic Review. Frontiers in Psychiatry, 9, 95.

Santana, B. M. Q., \& Ordi, H. G. (2016). Evaluación del" Mindfulness": aplicación del cuestionario" Mindfulness" de cinco facetas (FFMQ) en población española (Doctoral dissertation, Universidad Complutense de Madrid).

Singer, A. R., \& Dobson, K. S. (2007). An experimental investigation of the cognitive vulnerability to depression. Behaviour Research and Therapy, 45(3), 563-575.

Thompson, B. L., \& Waltz, J. (2010). Mindfulness and experiential avoidance as predictors of posttraumatic stress disorder avoidance symptom severity. Journal of Anxiety Disorders, 24(4), 409415.

Troy, A. S., Ford, B. Q., McRae, K., Zarolia, P., \& Mauss, I. B. (2016). Change the things you 
can: Effective emotion regulation is most beneficial in low socioeconomic contexts. Emotion, 17, 141-154.

Vujanovic, A. A., Youngwirth, N. E., Johnson, K. A., \& Zvolensky, M. J. (2009). Mindfulnessbased acceptance and posttraumatic stress symptoms among trauma-exposed adults without axis I psychopathology. Journal of Anxiety Disorders, 23(2), 297-303. 